\title{
Optical absorption studies in absorbing Bragg reflectors
}

\author{
J.L. Shen ${ }^{\mathrm{a}, *}$, C.Y. Chang ${ }^{\mathrm{a}}$, P.N. Chen ${ }^{\mathrm{a}}$, W.C. Chou ${ }^{\mathrm{a}}$, Y.F. Chen ${ }^{\mathrm{b}}$, M.C. Wu ${ }^{\mathrm{c}}$ \\ a Department of Physics, Chung Yuan Christian University, Chung-Li 320, Taiwan, ROC \\ b Department of Physics, National Taiwan University, Taipei 106, Taiwan, ROC \\ ${ }^{\mathrm{c}}$ Department of Electrical Engineering, University of California at Los Angeles, Los Angeles, CA 90095-1594, USA
}

Received 3 July 2001; accepted 26 September 2001

\begin{abstract}
Reflectivity and photoluminescence have been used to study optical absorption from an absorbing Bragg reflector consisting of AlAs/GaAs Bragg reflector and InGaAs/InGaAsP multiple-quantum-well absorbing layer. An absorption dip due to the optical confinement of the Fabry-Perot resonance was observed in the reflectivity spectrum. Based on the transfer matrix method along with the complex index of refraction, we successfully analyzed the reflectivity spectrum and obtained the absorption coefficient of the absorbing cavity around $1550 \mathrm{~nm}$. We also demonstrated that the quality factor of the Fabry-Perot mode in photoluminescence could be used to estimate the absorption coefficient of the cavity medium in our absorbing Bragg reflector. (c) 2001 Elsevier Science B.V. All rights reserved.
\end{abstract}

PACS: 77.55.+f; 78.40.-q; 78.55.-m

Keywords: Bragg reflector; Absorption

\section{Introduction}

Bragg reflectors, which consist of periodically alternating high and low index quarter-wavelength layers, are extensively used in many optoelectronic devices, such as vertical-cavity surface emitting lasers (VCSELs), microcavity light-emitting diodes (MCLEDs), and resonance-cavity photodetectors (RECAPs). Among these devices, VCSELs and MCLEDs usually require highly transparent dielectric layers for efficient operation and it is particularly important to eliminate any loss due to

\footnotetext{
${ }^{*}$ Corresponding author. Tel.: +886-3-4563171; fax: +886-34367981.

E-mail address: jlshen@phys.cycu.edu.tw (J.L. Shen).
}

the optical absorption in the Bragg reflectors. RECAPs, on the other hand, are designed to effectively increase the thickness of the absorption layer and thus enhance the light absorption in the active layer. Recently, absorbing media are also employed along with the layer-by-layer distribution of the refractive index to control the optical absorption in Bragg reflectors [1]. The optical absorption can then be enhanced or reduced according to the distribution both of the refractive index and of the absorbing media in the Bragg reflectors. Moreover, the saturable Bragg reflectors, which consist of semiconductor quantum wells embedded in quarter-wave stacks, are designed to enhance the optical absorption by the photon localization in a spectral range of the stop band. Subpicosecond pulses have been achieved 
using the saturable Bragg reflectors in the passively or hybrid mode locking for the solid state and semiconductor lasers [2,3]. Since the absorption effect is essential in such optoelectronic devices, it is desirable to investigate the fundamental properties of optical absorption in the Bragg reflectors.

In this report, we study the optical absorption of absorbing Bragg reflectors in the room-temperature reflectivity and photoluminescence (PL). A dip in the high-reflectance band (stop band) of the Bragg reflectors in reflectivity is attributed to the optical absorption from the Fabry-Perot cavity resonance. The measured reflectivity of the absorbing Bragg reflector was modeled using the transfer matrix method with a complex refractive index. The absorption coefficient of the cavity layer around $1550 \mathrm{~nm}$ is accordingly obtained from the calculations. In addition, the quality factor $(Q-$ factor) of the absorbing Bragg reflector was studied in the reflectivity and PL spectra. To the best of our knowledge, no measurement on the $Q$-factor of absorbing Bragg reflectors has been reported before. The absorption coefficient of the cavity layer is estimated from the $Q$-factor measurement. The discrepancy of the $Q$-factor between the reflectivity and PL measurements is explained by the re-absorption in PL due to the cavity resonance.

\section{Experiment}

The absorbing Bragg reflector studied here consisted of a GaAs/AlAs distributed Bragg reflector and an absorbing cavity layer. The distributed Bragg reflector, consisting of 27 pairs of GaAs/AlAs quarter-wave stacks, was used to increase the reflectivity and bandwidth of the reflector. The dielectric layers were designed for maximum reflectivity at $1550 \mathrm{~nm}$ at normal incidence. The absorbing cavity layer had two sets of $15 \mathrm{InGaAs} / \mathrm{InGaAsP}$ strain-compensated multiple quantum wells separated by $80 \mathrm{~nm}$ of lattice matched InGaAsP and grown on an InP substrate. Fig. 1 shows a schematic diagram of the absorbing Bragg reflector studied in this work. In this structure, the optical field in the cavity layer (curve in Fig. 1) is increased due to the cavity confine-

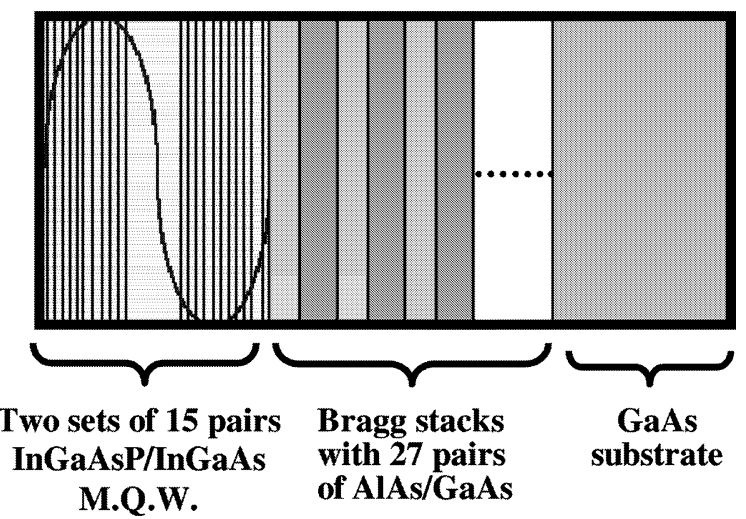

Fig. 1. Schematic diagram of the absorbing Bragg reflector studied in this work. The optical field in the cavity, calculated by transfer matrix method, is shown as a curve.

ment, leading to the enhancement of the optical absorption. The absorption properties of the sample were characterized using reflectivity and PL spectra at normal incidence at room temperature. The reflected signals were taken with a halogen lamp in conjunction with a monochromator. The PL was measured using a focused Ar ion laser as the excitation source and analyzed with a grating spectrometer. A germanium photodetector was used to read both the reflectivity and PL signals.

\section{Results and discussion}

Fig. 2 shows the measured reflectivity spectrum of the absorbing Bragg reflector. The spectrum is a typical reflectivity of Bragg reflector, but including an extra large dip at $1545 \mathrm{~nm}$ in the stop band. From the reflectivity measurement, we obtain the wavelength of the stop band center $(\lambda)$ in the Bragg reflectors, which is determined by the optical thickness $d$ of the Bragg stacks $\left(d=\lambda_{\text {air }} / 4 n=\right.$ $\lambda / 4$, where $n$ is the refractive index; $\lambda_{\text {air }}$ and $\lambda$ are the wavelength of light in the free space and the layered material, respectively). To explain the behavior of the extra dip in the reflectivity spectrum, we calculate the reflectivity using the matrix formulation for multi-layer structures [4]:

$$
\left(\begin{array}{ll}
M_{11} & M_{12} \\
M_{21} & M_{22}
\end{array}\right)=D_{0}^{-1} D_{\mathrm{c}} P_{\mathrm{c}} D_{\mathrm{c}}^{-1}\left[D_{\mathrm{L}} P_{\mathrm{L}} D_{\mathrm{L}}^{-1} D_{\mathrm{H}} P_{\mathrm{H}} D_{\mathrm{H}}^{-1}\right]^{27} D_{\mathrm{H}}
$$




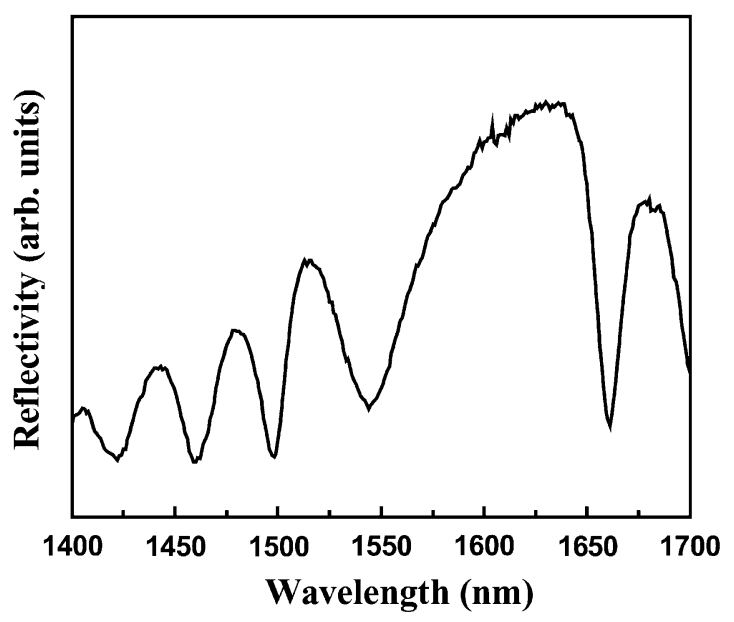

Fig. 2. Measured reflection spectrum of absorbing Bragg reflectors at room temperature.

where $D_{0}, D_{\mathrm{c}}, D_{\mathrm{H}}$, and $D_{\mathrm{L}}$ are the dynamic matrices for free space, cavity layer, GaAs, and AlAs, respectively; $P_{\mathrm{c}}, P_{\mathrm{H}}$, and $P_{\mathrm{L}}$ are the propagation matrices for the cavity layer, GaAs, and AlAs, respectively. Fig. 3(a) (dashed line) displays the theoretical reflectivity $R=\left|M_{21} / M_{11}\right|^{2}$ without considering the absorption in the cavity layer. As can be seen, the simulated result reproduces the char-

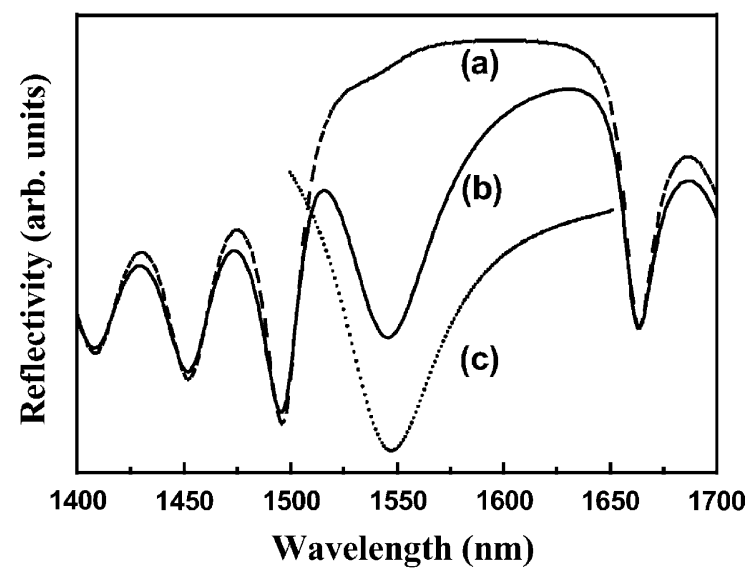

Fig. 3. Calculated reflection spectra of absorbing Bragg reflector. (a) The absorbing layer in the structure is described by a real index of refraction. (b) The absorbing layer in the structure is described by a complex index of refraction with an imaginary part $\kappa=0.049$. (c) The difference spectrum in stop-band region is obtained by subtracting (a) from (b). acteristics of the stop band and side lobes, but it shows a remarkable disagreement with the dip at $1545 \mathrm{~nm}$. The theoretical calculations can be improved by introducing an absorption (a loss term) in the cavity layer. To include the optical absorption, an imaginary part of the index of refraction $(\kappa)$ for the cavity layer is introduced for a loss in the reflectivity. As the loss in the cavity layer is included, the calculated reflectivity produces a dip in the stop band. The imaginary part of the index of refraction was used as an adjustable parameter to fit the measured data until the calculated and experimental reflectivity agreed. Fig. 3(b) (solid line) shows the theoretical reflectivity under the consideration of the absorption in the cavity layer. Comparing Fig. 3(b) to Fig. 2, the calculated result well reproduces all the important features in the experiment. According to the above analysis, the large dip in the stop band is strongly related to the cavity layer. We suggest that the dip is due to the optical confinement of the Fabry-Perot resonance at the cavity layer, which is sandwiched by the air/cover-layer interface and the Bragg mirror. In Fig. 3(b), a value of $\kappa=0.049$ is obtained from the fit of the dip in the stop band. Accordingly, the absorption coefficient of the cavity layer $(\alpha)$ can also be given by the following expression: $\alpha=$ $4 \pi \kappa / \lambda_{\text {air }}$. Taking into account $\kappa=0.049$ and $\lambda_{\text {air }}=1545 \mathrm{~nm}$, we obtain $\alpha=3985 \mathrm{~cm}^{-1}$ at 1545 $\mathrm{nm}$, which is close to the literature value [5].

In addition to the reflectivity studies, PL also provides another way to investigate the absorption of the absorbing Bragg reflector. Fig. 4 shows the PL spectrum of the sample performed at normal incidence. Compared with the spectrum in Fig. 2 the main peak occurred at $1548 \mathrm{~nm}$ in the PL corresponds to the dip in the reflectivity, caused by the Fabry-Perot cavity mode at the cavity layer. To confirm the assignment that the PL is due to the cavity mode, the energy shift of the peak owing to the thermal effect was estimated. It is known that the energy shift with varying temperature is $\sim 3.3 \mathrm{~A} /{ }^{\circ} \mathrm{C}$ for the InGaAs quantum-well transition and $\sim 0.85 \mathrm{~A} /{ }^{\circ} \mathrm{C}$ for the cavity mode in a similar structure [6]. As the temperature is decreased, the main peak shifts toward the shorter wavelength at a rate of $0.78 \mathrm{~A} /{ }^{\circ} \mathrm{C}$ (not shown here). Therefore, the peak at $1548 \mathrm{~nm}$ is attributed 


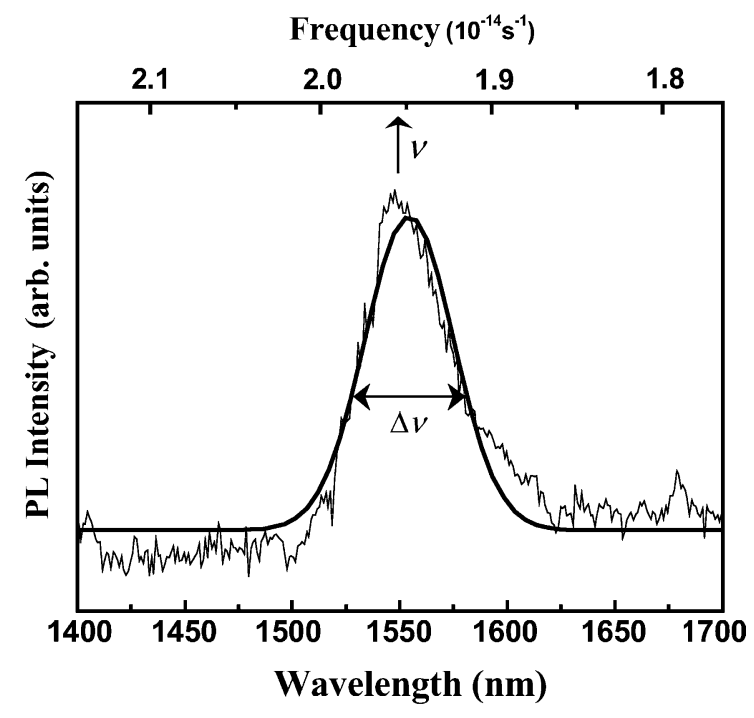

Fig. 4. The PL spectrum of absorbing Bragg reflector.

to be the luminescence due the Fabry-Perot mode of the cavity layer. The PL spectrum can be used to estimate the absorption coefficient of the cavity medium by the $Q$-factor. The $Q$-factor $Q$ of a cavity, which measures the sharpness of the resonance, is given by [7]:

$$
\begin{aligned}
Q & =v / \Delta v \\
& =2 \pi n_{\mathrm{c}} v\left[c\left(\alpha-\left(1 / L_{\mathrm{eff}}\right) \ln \left(R_{1} R_{2}\right)^{1 / 2}\right)\right]^{-1},
\end{aligned}
$$

where $v$ is the resonant frequency of the cavity, $\Delta v$ is the full width at half maximum (FWHM), $n_{\mathrm{c}}$ is the index of refraction of cavity layer, $\alpha$ is the absorption coefficient of the cavity medium, $L_{\text {eff }}$ is the effective cavity length, and $R_{1}$ and $R_{2}$ are the mirror reflectivity on each cavity side. To calculate the $Q$ factor of the Fabry-Perot mode, the PL signal was analyzed using a Gaussian profile (see the bold solid line in Fig. 4). In this analysis, a resonant frequency $v=1.93 \times 10^{14} \mathrm{~s}^{-1}$ and a FWHM $\Delta v=$ $6.4 \times 10^{12} \mathrm{~s}^{-1}$ is obtained, and the measured $Q$ is thus $\sim 30.2$. The effective cavity length $L_{\text {eff }}$ actually includes the cavity length $\left(L_{\mathrm{c}}\right)$ and the penetration of the cavity field into the GaAs/AlAs Bragg mirror ( $\left.L_{\mathrm{DBR}}\right)$, which can be estimated by the formula [8] $L_{\mathrm{DBR}}=\left[\lambda /\left(2 n_{\mathrm{c}}\right)\right]\left(n_{\mathrm{L}} n_{\mathrm{H}}\right) /\left(n_{\mathrm{H}}-n_{\mathrm{L}}\right)$. By taking $R_{1} \sim$ 0.99 (the estimated reflectivity of AlAs/GaAs Bragg mirror), $R_{2} \sim\left[\left(n_{\mathrm{c}}-1\right) /\left(n_{\mathrm{c}}+1\right)\right]^{2} \sim 0.32$ (the esti- mated reflectivity of cavity layer/air interface), $L_{\text {eff }}=L_{\mathrm{c}}+L_{\mathrm{DBR}} \sim 6663 \mathrm{~nm}$, and $n_{\mathrm{c}} \sim 3.6$ (the measured value from the angle-dependent PL) into the Eq. (2), we obtain $\alpha \sim 3951 \mathrm{~cm}^{-1}$. This absorption coefficient is close to the literature value and consistent with previous fitted value $\left(\alpha \sim 3985 \mathrm{~cm}^{-1}\right)$, obtained from the transfer matrix calculation. Thus, we demonstrated that the PL could be used as a convenient tool to study the absorption coefficient of the cavity layer.

By measuring the FWHM of the Fabry-Perot mode in the stop band, the $Q$-factor of the absorbing Bragg reflector can also be estimated in the reflectivity spectrum. However, the exact line width of the Fabry-Perot mode in Fig. 2 is ambiguous. To clearly find out the FWHM of FabryPerot mode in reflectivity, a difference spectrum was computed by subtracting Fig. 3(a) from Fig. 3(b). The difference spectrum in the stop-band region is shown in Fig. 3(c), where the FWHM due to the cavity mode is estimated to be $\sim 6.8 \times 10^{12}$ $\mathrm{s}^{-1}$. Accordingly, the $Q$-factor from the reflectivity spectrum is thus $\sim 28.4$. It is notable that the $Q$-factor obtained from reflectivity is smaller than that from PL. Same behavior has been found in the VCSEL and microcavity structures [6,9]. The larger $Q$-factor for PL can be explained by the fact that the PL is often modified by the re-absorption due to the cavity resonance. As the resonance effect is enhanced, the re-absorption increases and leads to line narrowing of the PL peak. This explanation is in agreement with the experimental results in Ref. [9], which shows the line width narrowing is pronounced as the pair number of Bragg reflectors is increased.

\section{Conclusion}

In conclusion, optical absorption of an absorbing Bragg reflector was studied by reflectivity and PL. An absorption dip originated from the Fabry-Perot cavity mode was observed in the reflectivity spectrum. It has been shown that the reflectivity including the absorption dip can be analyzed as the index of refraction is assumed to have an imaginary part. From the fit of absorption dip we were able to obtain the absorption coeffi- 
cient of the cavity layer around $1550 \mathrm{~nm}$, which is close to the literature value. In addition, the $Q$-factor of the absorbing Bragg reflector was studied in reflectivity and PL spectra. We have proposed the possibility for using the $Q$-factor to estimate the absorption coefficient of the cavity medium. The discrepancy of the $Q$-factor between the reflectivity and PL measurement is explained by the re-absorption in the PL due to the cavity resonance.

\section{Acknowledgements}

This project was supported in part by the National Science Council under the grant numbers 89-2112-M-033-006.

\section{References}

[1] K. Ogawa, Y. Matsui, T. Itatani, K. Ouchi, Appl. Phys. Lett. 72 (1998) 155.

[2] J.L. Shen, T. Jung, S. Murthy, T. Chau, M.C. Wu, Y.H. Lo, C.L. Chua, Z.H. Zhu, J. Opt. Soc. Am. B 16 (1999) 1064.

[3] S. Tsuda, W.H. Knox, S.T. Cundiff, W.Y. Jan, J.E. Cunningham, IEEE J. Select. Topics Quant. Electron. 2 (1996) 454.

[4] P. Yeh, Optical Waves and Layered Media, Wiley, New York, 1991.

[5] E. Zielinski, H. Schweizer, K. Streubel, H. Eisele, G. Weimann, J. Appl. Phys. 59 (1986) 2196.

[6] G.M. Yang, M.H. MacDougal, H. Zhao, P.D. Kapkus, J. Appl. Phys. 78 (1995) 3605.

[7] A. Yariv, Optical Electronics in Modern Communication, Oxford, New York, 1997.

[8] V. Savona, L.C. Andreani, P. Schwendimann, A. Quattropani, Solid State Commun. 93 (1995) 733.

[9] Y. Hanamaki, H. Akiyama, Y. Shjraki, Semicond. Sci. Technol. 14 (1999) 797. 